\section{Body composition assessment using DXA in six-year-old children: the 2004 Pelotas Birth Cohort, Rio Grande do Sul State, Brazil}

\author{
Composição corporal avaliada por DXA aos seis \\ anos de idade: Coorte de Nascimentos de Pelotas \\ de 2004, Rio Grande do Sul, Brasil
}

\section{La composición corporal evaluada por DXA a los seis años de edad: cohorte de nacimientos en Pelotas, 2004, Río Grande do Sul, Brasil}

\begin{abstract}
The aim of this study was to describe fat (FM) and lean body mass (LBM) in six-year-old children from the 2004 Pelotas Birth Cohort, stratified by gender. Dual-Energy X-ray Absorptiometry was used to measure FM and LBM, FM and $L B M$ indexes, and percentage (\%) of FM and LBM. Mean measures of adiposity were higher among girls $\left(6.3 \mathrm{~kg}, 4.2 \mathrm{~kg} / \mathrm{m}^{2}\right.$ and $23.4 \%$ vs. $5 \mathrm{~kg}$, $3.3 \mathrm{~kg} / \mathrm{m}^{2}$ and $\left.18 \%\right)$ while LBM measures were higher among boys (19.3kg, $13 \mathrm{~kg} / \mathrm{m}^{2}$ and $78.5 \%$ vs. $17.7 \mathrm{~kg}, 12.2 \mathrm{~kg} / \mathrm{m}^{2}$ and $73.2 \%$ ). In both boys and girls mean measures of adiposity increased with socioeconomic status and maternal education. Mean measures of adiposity were higher among white-skinned children while \%LBM was higher among black-skinned children. Preterm compared to full-term children showed lower mean measures of adiposity and LBM. Female sex, white skin color and higher socioeconomic conditions are associated with higher adiposity in childhood.
\end{abstract}

Body Composition; Child; Longitudinal Studies
Roberta V. Zanini 1

Iná S. Santos 1

Denise P. Gigante 1

Alicia Matijasevich 1

Fernando C. Barros 1

Aluísio J. D. Barros 1

\section{Resumo}

O objetivo deste estudo foi descrever as massas gorda (MG) e magra (MM) em crianças de seis anos de idade na Coorte de Nascimentos de Pelotas de 2004, Rio Grande do Sul, Brasil, estratificadas por sexo. Dual-Energy X-ray Absorptiometry foi usado para medir $M G$ e $M M$, indices de $M G$ e MM e percentuais (\%) de $M G$ e MM. Médias de adiposidade foram maiores entre as meninas $\left(6,3 \mathrm{~kg}, 4,2 \mathrm{~kg} / \mathrm{m}^{2}\right.$ e 23,4\% vs. $5 \mathrm{~kg}, 3,3 \mathrm{~kg} / \mathrm{m}^{2}$ e $18 \%$ ), enquanto as medidas de MM foram maiores entre os meninos $(19,3 \mathrm{~kg}$, $13 \mathrm{~kg} / \mathrm{m}^{2}$ e $78,5 \%$ vs. $17,7 \mathrm{~kg}, 12,2 \mathrm{~kg} / \mathrm{m}^{2}$ e $73,2 \%$ ). Em meninos e meninas as médias de adiposidade aumentaram com o nível socioeconômico e escolaridade materna. Médias de adiposidade foram maiores entre crianças de cor branca, enquanto \%MM foi maior entre as de cor preta. Crianças pré-termo mostraram menores médias de adiposidade e MM, em comparação com nascidas a termo. Sexo feminino, cor branca e condições socioeconômicas mais elevadas estão associados à maior adiposidade na infância.

Composição Corporal; Criança; Estudos

Longitudinais 


\section{Introduction}

The assessment of body composition in children especially body fat measures has become a focus of scientific research for its ability to detect early changes that may potentially have longterm harmful health effects. Physical changes that occur rapidly in the postnatal period and throughout childhood and adolescence continue at a slower rate into adult life 1 . These changes are apparent both internally and externally throughout childhood and adolescence: body proportion, height, weight and pubertal stage can easily be assessed by means of physical examination and anthropometric measures. To assess internal changes including hormone levels and body composition, however, appropriated methods are required 2 . The approaches currently available for the assessment of body composition are categorized as direct (analysis of cadavers), indirect (densitometry, plethysmography, hydrodensitometry and underwater weighing) and doubly indirect (bioelectrical impedance and anthropometric measures) 3 .

The increase in prevalence of obesity, including in childhood, has led to a number of studies on the theme, mainly in developed and in European countries 4,5,6. In general, these studies showed that greater adiposity in infancy and childhood was associated to female sex 4,5,6,7,8,9, white skin color 7,9,10,11, higher birth 4,12 and current weight $12,13,14$, higher pre-gestational maternal 4,7,15 and paternal body mass index 14,16, maternal smoking in pregnancy $15,16,17$, and shorter duration of breastfeeding 6,18 , whereas the role of socioeconomic conditions is controversial at the literature $7,14,16,19,20$. No studies were found in countries such as Brazil, where epidemiological and nutritional transitions are still playing out 21,22 , leaving a gap with regard to the effect of these transitions on body composition in childhood. So, this study aimed to describe fat and lean body mass measurements in six-year-old children from the 2004 Pelotas Birth Cohort, in Southern Brazil.

\section{Subjects and methods}

Since the perinatal study in 2004 there were five follow-ups (at 3, 12, 24, 48 and 72 months) of all cohort subjects. Details on the study methods are available elsewhere 23 . The fifth follow-up was from October 2010 to August 2011 when the cohort children were six to seven years old. A total of 3,722 children ( $90.2 \%$ of the original cohort) were followed up, of which 3,556 (95.5\%) attended a visit at the study clinic. Of these, 3,437 underwent an assessment of body composition by Dual Energy X-ray Absorptiometry (DXA). Over two thirds of the children $(n=2519)$ were evaluated at the clinic while 1,037 were initially evaluated at home (when height and weight measures were taken) as they were not brought in by their mothers/ guardians after the study visit had been rescheduled more than three times. They then attended a visit at the clinic for the assessment of body composition. The sample studied consisted of 3,373 children excluding twins $(n=64)$. Outcome measures were obtained using a DXA with enCORE software platform (Lunar Prodigy, GE Healthcare, USA). The children wore light, fitted clothing (shorts and sleeveless shirts; no metal accessories) and a trained technician took all measurements with children in the supine position.

The dependent variables studied included fat mass (FM) and lean body mass (LBM) in kg; fat mass index (FMI) and lean body mass index (LBMI) in $\mathrm{kg} / \mathrm{m}^{2}$; and percentage of fat mass $(\% \mathrm{FM})$ and percentage of lean body mass (\%LBM). All measures in $\mathrm{kg}$ were adjusted for body weight obtained in a high precision scale $(0.01 \mathrm{~kg}$ resolution) (model BWB-627-A, Tanita Corporation, Japan, modified by Life Measurement, Inc., USA). This adjustment was calculated as follows:

$$
F M_{\text {ajusted }} \text { or } L B M_{\text {ajusted }}=\left(\frac{F M \text { or } L B M}{T B M}\right) \times \text { Weight }
$$

Where:

FM: fat mass directly provided by DXA (kg) LBM: lean mass directly provided by DXA $(\mathrm{kg})$ TBM: total body mass resulting from the sum of (FM + LM + bone mass), directly provided by DXA (kg)

Weight: obtained from a high precision scale $(\mathrm{kg})$

FMI and LBMI were calculated by dividing the adjusted FM or LBM by height in $\mathrm{m}^{2}$. FMI and LBMI were used because children can have different percentages of FM due to different absolute amounts of FM but equivalent amounts of fat free mass (FFM) or different absolute amounts of FFM and same amounts of FM. The normalization of these measures for height squared $(\mathrm{kg} /$ $\mathrm{m}^{2}$ ) may improve the sensitivity to detect changes in body composition measurements 24 . Height was measured at the clinic using a stadiometer with a precision of $1 \mathrm{~mm}$ and maximum capacity of 2.06m (Harpenden, Holtain, Crymych, UK), and at home using a stadiometer with a precision of $1 \mathrm{~mm}$ and maximum capacity of $2.13 \mathrm{~m}$ (Alturexata, Belo Horizonte, Brazil). At the clinic, two independent examiners took at least two height measures and the average of the two values was recorded, when the difference between the two measures was greater than $0.7 \mathrm{~cm} 25$ $(\mathrm{n}=73)$. The \%FM and \%LBM were directly provided by the equipment with no adjustment: FM 
or LBM divided by TBM, multiplied by 100 . Of 3,373 children, 71 did not have weight measures in a high precision scale, 38 did not have height measures and seven did not have either weight or height measures. Thus, there was FM or LBM data for 3,295 children and FMI or LBMI data for 3,257 children.

The independent variables were derived from information collected in the perinatal study. The socioeconomic index was constructed through principal component analysis based on information about consumer goods and education of the head of the family 26 divided into five socioeconomic quintiles for the city of Pelotas (Q1 lower; Q5 upper). Maternal education level was determined according to the number of full years at school. Gestational age was obtained from the algorithm as proposed by the National Center for Health Statistics (NCHS) 27. It was estimated from the last menstrual period date (LMPD) when this data was consistent with height, weight and head circumference measures at birth and the parameters of normal growth curves for each GA ${ }^{28}$. When LMPD was unknown or inconsistent, gestational age was estimated from ultrasound examination (up to 20 weeks) if available or from the Dubowitz clinical maturity score calculated in all newborns 29 . Data on skin color of the child was collected at age six reported by the respondent of the questionnaire (usually the mother). Birth weight and length were obtained using electronic pediatric scales with a precision of $10 \mathrm{~g}$ and infantometers with a precision of $1 \mathrm{~mm}$ (Harpenden, Holtain, Crymych, UK), respectively.

The statistical analyses were performed using Stata version 12.0 (Stata Corp., College Station, USA). The analysis of variance was carried out to assess means and standard deviations (SDs) of each outcome by gender. Tests of linear trend and heterogeneity were used according to the variable category. Nonparametric tests were performed when the assumption of homogeneity has not been met. In addition, 95\% confidence intervals $(95 \% \mathrm{CI})$ were calculated. The quality of data collected during the fifth follow-up was ensured by a set of actions taken prior to and during the fieldwork including training of interviewers and equipment technicians, standard examination procedures and weekly meetings with the supervisor. During the perinatal study, data quality control included a small survey administered at home or in the hospital to check interview status 23 .

This study was approved by the Research Ethics Committee of the University of Pelotas Medical School. All mothers/guardians signed an informed consent form prior to the interview.

\section{Results}

The mean age of the 3,373 children followed up was $80.4 \pm 2.4$ months. At birth, $23 \%$ of these children were in the lower quintile of socioeconomic index and $43 \%$ of the mothers had nine years of formal education or more. More than two thirds of the mothers reported having white-skinned children. As for gestational age, $12.3 \%$ were preterm children (less than 37 weeks of gestation) (Table 1). In general, boys were bigger than girls at birth, they had greater birth weight $(\mathrm{p}<0.0001)$ and birth length $(\mathrm{p}<0.0001)$; this difference was also seen at age six for height $(p<0.0001)$, but not for weight $(\mathrm{p}=0.1$ ) (Table 2). Girls had higher mean adiposity measures (FM, \%FM and FMI) compared to boys while boys had higher mean LBM. FM range (minimum and maximum) was similar in boys and girls but girls showed on average an excess $1.3 \mathrm{~kg}$ of fat compared to boys. After adjusting for height, this difference was smaller with a mean value of $0.9 \mathrm{~kg} / \mathrm{m}^{2}$ greater in girls. Regarding the percentage of body fat, a 5.4 higher percentage was seen in girls compared to boys (Table 2). The range of LBM and LBMI was similar in both boys and girls, but boys had higher means $\left(1.6 \mathrm{~kg}\right.$ and $0.8 \mathrm{~kg} / \mathrm{m}^{2}$ higher, respectively) than girls. As for \%LBM, boys showed greater minimum and maximum values that were on average 5.3\% higher than those from girls (Table 2). Weight and height measures in boys and girls age six were positively associated with maternal education levels and socioeconomic index. With respect to skin color, there was a difference in mean height in boys. Compared to full-term children (37 to 41 weeks of gestation), preterm children or those born at 41 weeks of gestation or more were shorter (Table 3).

Mean FM, \%FM and FMI in both girls (Table 4), and boys (Table 5) increased with socioeconomic index and maternal education level. Children who were poor at birth had lower FM at age six compared to those who were in a more advantageous position. Among girls, greater differences were observed between Q1 and Q4; those in the upper position showed on average $2.1 \mathrm{~kg}$, $4.9 \%$ and $1.1 \mathrm{~kg} / \mathrm{m}^{2}$ higher FM, \%FM and FMI, respectively, than those in the lower one. Among boys, greater differences were seen between Q1 and Q5; those in the upper position had on average $3.0 \mathrm{~kg}, 7.8 \%$ and $1.7 \mathrm{~kg} / \mathrm{m}^{2}$ higher FM, \%FM and FMI, respectively, than those in the lower one. Mean LBM increased in the same direction while \%LBM showed an inverse association, with greater values among poor children and those born to less educated mothers. In general, mean adiposity measures were higher among white children with a statistically significant differ- 
Description of the study independent variables, overall and by gender. The 2004 Pelotas Birth Cohort, Pelotas, Rio Grande do Sul State, Brazil, 2013. ( $N=3,373)$.

\begin{tabular}{|c|c|c|c|c|c|c|}
\hline \multirow[t]{2}{*}{ Variables } & \multicolumn{2}{|c|}{ Girls } & \multicolumn{2}{|c|}{ Boys } & \multicolumn{2}{|c|}{ Overall } \\
\hline & $\mathbf{n}$ & $\%$ & $\mathbf{n}$ & $\%$ & $\mathrm{n}$ & $\%$ \\
\hline \multicolumn{7}{|c|}{ Socioeconomic Index } \\
\hline Q1 (20-280) & 375 & 23.1 & 395 & 22.7 & 770 & 22.9 \\
\hline Q2 (281-367) & 356 & 21.9 & 357 & 20.6 & 713 & 21.2 \\
\hline Q3 (368-475) & 368 & 22.7 & 390 & $22, .5$ & 758 & 22.6 \\
\hline Q4 (476-618) & 260 & 16.0 & 291 & 16.8 & 551 & 16.4 \\
\hline Q5 (619-999) & 264 & 16.3 & 304 & 17.5 & 568 & 16.9 \\
\hline \multicolumn{7}{|c|}{ Maternal education (years) } \\
\hline $0-4$ & 244 & 15.2 & 260 & 15.0 & 504 & 15.1 \\
\hline $5-8$ & 686 & 42.6 & 714 & 41.2 & 1,400 & 41.9 \\
\hline $9-11$ & 543 & 33.7 & 578 & 33.4 & 1,121 & 33.5 \\
\hline 12 or more & 138 & 8.6 & 180 & 10.4 & 318 & 9,5 \\
\hline \multicolumn{7}{|l|}{ Skin color } \\
\hline White & 1,094 & 67.7 & 1,150 & 67.1 & 2,244 & 67,4 \\
\hline Black & 186 & 11.5 & 231 & 13.5 & 417 & 12,5 \\
\hline Brown & 237 & 14,7 & 252 & 14.7 & 489 & 14,7 \\
\hline Other & 99 & 6.1 & 82 & 4.8 & 181 & 5,4 \\
\hline \multicolumn{7}{|c|}{ Gestational age (weeks) } \\
\hline$<34$ & 32 & 2.0 & 39 & 2.2 & 71 & 2,1 \\
\hline $34-36$ & 171 & 10.5 & 174 & 10.0 & 345 & 10,2 \\
\hline $37-41$ & 1,318 & 80.9 & 1,421 & 81.6 & 2,739 & 81,3 \\
\hline$>41$ & 108 & 6.6 & 108 & 6.2 & 216 & 6,4 \\
\hline
\end{tabular}

* Minimum and maximum values of socioeconomic index score in each category.

ence in FM and FMI among boys and \%FM and FMI among girls. The \%LBM was greater among black-skinned children. Lower mean FM, \%FM, FMI and LBM were found in preterm children compared to those born at 37 to 41 weeks of gestation (Tables 4 and 5).

\section{Discussion}

The most important strengths of the current study lie in the use of population-based data, low loss to follow-up rates and use of DXA, a valid method for the assessment of body composition in children 30 . The main weakness of this study was the fact that anthropometric measures taken in different settings could have resulted in different height measures. The equipment used and number of measurements was not the same at the clinic and at home. However, a comparison of mean heights of children taken at the clinic and at home showed similar measures in both boys $(\mathrm{p}=0.8)$ and girls $(\mathrm{p}=0.3)$.
Several studies using DXA assessed children across a wide age range and therefore presented their results as total estimates or by age group but not at individual ages 5,10,31,32. The analysis of mean FM and LBM including children across a wide age range in the same group makes it difficult to compare our results to those of other studies since the measurements of body composition vary substantially throughout childhood 2,33 . Yet, a review of the literature showed that, throughout childhood (age 0 to 9 years) and in many different countries, girls had higher mean adiposity measures than boys, while boys showed greater mean LBM and LBMI 4,5,6,7,8,9. Our results support these previous findings.

Mean \%FM among boys and girls (respectively, $18 \%$ and $23.4 \%$ ) were equivalent to those observed among British children (boys at centile 75 and girls in between centiles 85 and 91) at six years of age 34 . Mean FM estimates (boys: $5.0 \pm$ $3.9 \mathrm{~kg}$ and girls: $6.3 \pm 4.2 \mathrm{~kg}$ ) found in this study were similar to those found in eight-year-old Chinese children with normal weight (boys: 5.2 
Means and standard deviations of anthropometric variables and body composition measures. The 2004 Pelotas Birth Cohort, Pelotas, Rio Grande do Sul State, Brazil, 2013. ( $N=3,373)$.

\begin{tabular}{|c|c|c|c|c|c|c|c|c|c|}
\hline \multirow[t]{2}{*}{ Variable (unit) } & \multicolumn{4}{|c|}{ Girls } & \multicolumn{4}{|c|}{ Boys } & \multirow[t]{2}{*}{ p-value } \\
\hline & $\mathbf{n}$ & Mean \pm SD & Minimum & Maximum & $\mathbf{n}$ & Mean \pm SD & Minimum & Maximum & \\
\hline Birth weight (g) & 1,630 & $3,140.9 \pm 497.3$ & 900 & 5,265 & 1,743 & $3,247.5 \pm 522.5$ & 950 & 5,995 & 0.0001 * \\
\hline Birth height (cm) & 1,624 & $47.9 \pm 2.4$ & 27.5 & 55.8 & 1,736 & $48.7 \pm 2.5$ & 35.0 & 58.8 & $<0.0001$ ** \\
\hline Weight ${ }^{\star \star \star}$ at age $6(\mathrm{~kg})$ & 1,595 & $24.3 \pm 6.0$ & 13.6 & 58.0 & 1,700 & $24.7 \pm 5.8$ & 14.3 & 60.4 & 0.02 ** \\
\hline Weight \# at age 6 (kg) & 1,595 & $24.8 \pm 6.1$ & 14.2 & 58.9 & 1,700 & $25.1 \pm 5.8$ & 14.7 & 60.8 & $0.1 * \star$ \\
\hline Height at age $6(\mathrm{~m})$ & 1,612 & $1.20 \pm 0.06$ & 1.04 & 1.41 & 1,716 & $1.22 \pm 0.06$ & 1.05 & 1.45 & $<0.0001$ ** \\
\hline $\mathrm{FM} \# \#(\mathrm{~kg})$ & 1,595 & $6.3 \pm 4.2$ & 0.9 & 29.1 & 1,700 & $5.0 \pm 3.9$ & 0.8 & 29.4 & 0.0001 * \\
\hline FMI \#\# $\left(\mathrm{kg} / \mathrm{m}^{2}\right)$ & 1,578 & $4.2 \pm 2.5$ & 0.7 & 17.6 & 1,679 & $3.3 \pm 2.3$ & 0.6 & 16.4 & $0.0001 *$ \\
\hline \%FM (\%) & 1,630 & $23.4 \pm 9.4$ & 5.9 & 53.0 & 1,743 & $18.0 \pm 9.2$ & 4.5 & 50.2 & $<0.0001$ ** \\
\hline FM \#\#\# (kg) & 1,595 & $6.2 \pm 4.1$ & 0.8 & 28.7 & 1,700 & $4.9 \pm 3.9$ & 0.8 & 29.0 & $0.0001 *$ \\
\hline FMI \#\#\# (kg/m²) & 1,578 & $4.1 \pm 2.5$ & 0.7 & 17.4 & 1,679 & $3.2 \pm 2.3$ & 0.5 & 16.3 & 0.0001 * \\
\hline LM \#\# (kg) & 1,595 & $17.7 \pm 2.3$ & 12.1 & 28.4 & 1,700 & $19.3 \pm 2.3$ & 13.2 & 30.0 & 0.0001 ** \\
\hline LMI \#\# (kg/m²) & 1,578 & $12.2 \pm 0.9$ & 9.9 & 17.2 & 1,679 & $13.0 \pm 0.9$ & 10.3 & 17.0 & 0.0001 ** \\
\hline$\%$ LM (\%) & 1,630 & $73.2 \pm 9.1$ & 44.2 & 90.8 & 1,743 & $78.5 \pm 9,0$ & 46.9 & 91.7 & $<0.0001$ ** \\
\hline LM \#\# (kg) & 1,595 & $17.3 \pm 2.3$ & 11.6 & 27.9 & 1,700 & $19.0 \pm 2.4$ & 12.9 & 29.8 & $<0.0001$ ** \\
\hline LMI \#\#\# (kg/m²) & 1,578 & $11.9 \pm 0.9$ & 9.4 & 16.9 & 1,679 & $12.8 \pm 0.9$ & 10.0 & 16.8 & $<0.0001$ ** \\
\hline
\end{tabular}

FM: fat mass; FMI: fat mass index; \%FM: percentage of fat mass; LM: lean mass; LMI: lean mass index; \%LM: percentage of lean mass.

* Test of homogeneity (non-parametric);

** Test of homogeneity (parametric);

*** Sum of parts from DXA;

\# From high precision scale;

\#\# Adjusted for high precision scale;

\#\#\# Directly provided by DXA.

$\pm 1.6 \mathrm{~kg}$; girls: $6.2 \pm 1.9 \mathrm{~kg}$ ) ( $\mathrm{p}=0.9$ for both boys and girls) 35 . However, in comparison with Chinese children at the age of seven, children from the Pelotas cohort showed mean measures $1.5 \mathrm{~kg}$ and $2.5 \mathrm{~kg}$ higher in boys and girls, respectively 35 . Mean FMIs in our study were similar to those reported in Chinese children age seven and eight, except for girls who showed higher FMI at age seven in Pelotas 35.

A study with Korean children 8 found lower mean FMs in both boys and girls age six to seven compared to our results. FMs were $3.1 \mathrm{~kg}$ and $3.5 \mathrm{~kg}$ lower in Korean boys and girls, respectively, compared to mean values found in the Pelotas children. When children aged seven were compared, this difference became less remarkable, decreasing to $1.6 \mathrm{~kg}$ in boys and $2.7 \mathrm{~kg}$ in girls 8 . Mean LBM, \%LBM and LBMI were higher in boys than girls, which is consistent with findings from other studies 6, 8, 9, 19 .

Mean LBM measures found in Pelotas children (boys: $19.3 \pm 2.3 \mathrm{~kg}$; girls: $17.7 \pm 2.3 \mathrm{~kg}$ ) were similar to those reported in Korean children aged six (boys: $19.4 \pm 1.4 \mathrm{~kg}$; girls: $17.9 \pm 1.7 \mathrm{~kg}$ ) ${ }^{8}$. A New
Zealand study with children ${ }^{9}$ with mean age of eight among boys and mean age of seven among girls reported slightly higher means (boys: $21.8 \pm$ 5.5kg; girls: $18.8 \pm 3.7 \mathrm{~kg}$ ) compared to our study. However, after adjusting for height (boys: $13.1 \pm$ $0.9 \mathrm{~kg} / \mathrm{m}^{2}$; girls: $12.3 \pm 0.7 \mathrm{~kg} / \mathrm{m}^{2}$ ), these means were close to those found in Pelotas (boys: 13.0 $\pm 0.9 \mathrm{~kg} / \mathrm{m}^{2}$; girls: $\left.12.2 \pm 0.9 \mathrm{~kg} / \mathrm{m}^{2}\right)^{9}$. Nevertheless, population data should be interpreted with caution, as there are no reference curves for body composition measurements obtained by DXA. The differences in means across countries could be associated with genetic and environmental factors.

White boys had higher mean FM, \%FM and FMI compared to all other categories, with a larger difference seen between white and black children. These findings are in agreement with the literature; studies conducted in different settings with children at different stages of development found higher mean FM among white compared with black children 7,10,11. Among girls, no statistically significant difference in mean FM was seen according to skin color. White girls 
Mean weight and height in children at age six, according to independent variables. The 2004 Pelotas Birth Cohort, Pelotas, Rio Grande do Sul State, Brazil, 2013. $(N=3,373)$.

\begin{tabular}{|c|c|c|c|c|c|c|c|c|}
\hline \multirow[t]{2}{*}{ Variables } & \multicolumn{4}{|c|}{ Girls } & \multicolumn{4}{|c|}{ Boys } \\
\hline & $\mathrm{n}$ & Weight & $\mathrm{n}$ & Height & $\mathbf{n}$ & Weight & $\mathrm{n}$ & Height \\
\hline Socioeconomic index & & $p<0.0001 *$ & & $p<0.0001$ ** & & $p<0.0001$ * & & $p<0.0001$ ** \\
\hline Q1 (lower) & 366 & $\begin{array}{c}23.3 \\
(22.7,23.9)\end{array}$ & 367 & $\begin{array}{c}1.19 \\
(1.18,1.19)\end{array}$ & 380 & $\begin{array}{c}23.1 \\
(22.6,23.6)\end{array}$ & 387 & $\begin{array}{c}1.20 \\
(1.19,1.20)\end{array}$ \\
\hline Q2 & 351 & $\begin{array}{c}24.4 \\
(23.7,25.0)\end{array}$ & 351 & $\begin{array}{c}1.20 \\
(1.20,1.21)\end{array}$ & 344 & $\begin{array}{c}24.6 \\
(24.0,25.2)\end{array}$ & 349 & $\begin{array}{c}1.21 \\
(1.21,1.22)\end{array}$ \\
\hline Q3 & 361 & $\begin{array}{c}25.0 \\
(24.4,25.6)\end{array}$ & 367 & $\begin{array}{c}1.20 \\
(1.20,1.21)\end{array}$ & 385 & $\begin{array}{c}25.4 \\
(24.8,26.0)\end{array}$ & 383 & $\begin{array}{c}1.22 \\
(1.12,1.22)\end{array}$ \\
\hline Q4 & 255 & $\begin{array}{c}26.5 \\
(25.6,27.3)\end{array}$ & 258 & $\begin{array}{c}1.22 \\
(1.21,1.23)\end{array}$ & 288 & $\begin{array}{c}25.9 \\
(25.2,26.6)\end{array}$ & 289 & $\begin{array}{c}1.23 \\
(1.22,1.23)\end{array}$ \\
\hline Q5 (upper) & 255 & $\begin{array}{c}26.0 \\
(25.2,26.7)\end{array}$ & 262 & $\begin{array}{c}1.22 \\
(1.21,1.22)\end{array}$ & 297 & $\begin{array}{c}27.5 \\
(26.8,28.2)\end{array}$ & 302 & $\begin{array}{c}1.24 \\
(1.23,1.24)\end{array}$ \\
\hline Maternal education (years) & & $p<0.0001 * *$ & & $p<0.0001 * \star$ & & $p<0.0001 *$ & & $\mathrm{p}<0.0001$ ** \\
\hline $0-4$ & 238 & $\begin{array}{c}23.6 \\
(22.8,24.5)\end{array}$ & 240 & $\begin{array}{c}1.19 \\
(1.18,1.19)\end{array}$ & 255 & $\begin{array}{c}23.2 \\
(22.6,23.8)\end{array}$ & 256 & $\begin{array}{c}1.20 \\
(1.19,1.20)\end{array}$ \\
\hline $5-8$ & 673 & $\begin{array}{c}24.4 \\
(23.9,24.8)\end{array}$ & 681 & $\begin{array}{c}1.20 \\
(1.19,1.20)\end{array}$ & 690 & $\begin{array}{c}24.4 \\
(24.0,24.8)\end{array}$ & 698 & $\begin{array}{c}1.21 \\
(1.20,1.21)\end{array}$ \\
\hline $9-11$ & 530 & $\begin{array}{c}25.5 \\
(25.0,26.1)\end{array}$ & 535 & $\begin{array}{c}1.21 \\
(1.21,1.22)\end{array}$ & 570 & $\begin{array}{c}26.4 \\
(25.9,27.0)\end{array}$ & 573 & $\begin{array}{c}1.23 \\
(1.22,1.23)\end{array}$ \\
\hline 12 or more & 136 & $\begin{array}{c}26.6 \\
(25.6,27.6)\end{array}$ & 137 & $\begin{array}{c}1.22 \\
(1.21,1.23)\end{array}$ & 175 & $\begin{array}{c}26.7 \\
(25.8,27.5)\end{array}$ & 178 & $\begin{array}{c}1.24 \\
(1.23,1.24)\end{array}$ \\
\hline Skin color & & $p=0.6 * \star \star$ & & $p=0.7 \star \star \star$ & & $p=0.1 \star \star \star$ & & $p=0.04 * \star \star$ \\
\hline White & 1,069 & $\begin{array}{c}25.0 \\
(24.6,25.3)\end{array}$ & 1083 & $\begin{array}{c}1.20 \\
(1.20 ; 1,21)\end{array}$ & 1122 & $\begin{array}{c}25.4 \\
(25.0,25.7)\end{array}$ & 1135 & $\begin{array}{c}1.22 \\
(1.21,1.22)\end{array}$ \\
\hline Black & 183 & $\begin{array}{c}24.4 \\
(23.5,25.3)\end{array}$ & 183 & $\begin{array}{c}1.21 \\
(1.20 ; 1,21)\end{array}$ & 225 & $\begin{array}{c}24.8 \\
(24.0,25.6)\end{array}$ & 226 & $\begin{array}{c}1.22 \\
(1.21,1.22)\end{array}$ \\
\hline Brown & 231 & $\begin{array}{c}24.6 \\
(23.8,25.3)\end{array}$ & 234 & $\begin{array}{c}1.20 \\
(1.20,1.21)\end{array}$ & 245 & $\begin{array}{c}24.7 \\
(23.9,25.4)\end{array}$ & 247 & $\begin{array}{c}1.21 \\
(1.20,1.21)\end{array}$ \\
\hline Other & 98 & $\begin{array}{c}24.6 \\
(23.4,25.8)\end{array}$ & 98 & $\begin{array}{c}1.20 \\
(1.19,1.21)\end{array}$ & 81 & $\begin{array}{c}24.5 \\
(23.3,25.6)\end{array}$ & 81 & $\begin{array}{c}1.21 \\
(1.20,1.23)\end{array}$ \\
\hline Gestational age (weeks) & & $p=0.002 \#$ & & $p=0.0001 * \star \star$ & & $\mathrm{p}<0.0001^{\star \star \star}$ & & $p=0.0002 * \star *$ \\
\hline$<34$ & 32 & $\begin{array}{c}23.0 \\
(21.1,24.9)\end{array}$ & 31 & $\begin{array}{c}1.17 \\
(1.15,1.19)\end{array}$ & 36 & $\begin{array}{c}22.5 \\
(20.5,24.5)\end{array}$ & 39 & $\begin{array}{c}1.19 \\
(1.17,1.21)\end{array}$ \\
\hline $34-36$ & 169 & $\begin{array}{c}23.5 \\
(22.7,24.2)\end{array}$ & 168 & $\begin{array}{c}1.19 \\
(1.18,1.20)\end{array}$ & 172 & $\begin{array}{c}23.8 \\
(22.9,24.7)\end{array}$ & 171 & $\begin{array}{c}1.21 \\
(1.20,1.22)\end{array}$ \\
\hline $37-41$ & 1287 & $\begin{array}{c}25.1 \\
(24.8,25.5)\end{array}$ & 1304 & $\begin{array}{c}1.21 \\
(1.20,1.21)\end{array}$ & 1386 & $\begin{array}{c}25.5 \\
(25.1,25.8)\end{array}$ & 1399 & $\begin{array}{c}1.22 \\
(1.22,1.22)\end{array}$ \\
\hline$>41$ & 106 & $\begin{array}{c}24.2 \\
(23.2,25.3)\end{array}$ & 108 & $\begin{array}{c}1.20 \\
(1.18,1.21)\end{array}$ & 105 & $\begin{array}{c}24.3 \\
(23.3,25.3)\end{array}$ & 106 & $\begin{array}{c}1.20 \\
(1.19,1.22)\end{array}$ \\
\hline
\end{tabular}

* Test for linear trend (non-parametric);

** Test for linear trend (parametric);

$\star \star \star$ Test of homogeneity (parametric);

\# Test of homogeneity (non-parametric).

showed higher mean \%FM and FMI, with a larger difference observed between white and black girls, which corroborate the findings of other studies $7,10,11$.
The highest means \%LBMs was seen among black boys and girls. Studies that measured LBM using DXA found marked differences throughout childhood between white and black children, 
Mean fat mass (FM) and mean lean body mass (LBM) in girls, according to the study independent variables. The 2004 Pelotas Birth Cohort, Pelotas, Rio Grande do Sul State, Brazil, 2013. ( $N=3,373)$.

\begin{tabular}{|c|c|c|c|c|c|c|c|c|c|}
\hline \multirow[t]{2}{*}{ Variables } & \multicolumn{3}{|c|}{$\mathrm{kg}$} & \multicolumn{3}{|c|}{$\%$} & \multicolumn{3}{|c|}{$\mathrm{kg} / \mathrm{m}^{2}$} \\
\hline & $\mathbf{n}$ & $\mathrm{FM}$ & LM & $\mathbf{n}$ & FM & LM & $\mathbf{n}$ & FM & LM \\
\hline Socioeconomic index & & $\mathrm{p}<0.0001$ * & $p<0.0001 * \star$ & & $\mathrm{p}<0.0001 * \star$ & $\mathrm{p}<0.0001 \star \star$ & & $\mathrm{p}<0.0001$ ** & $p=0,8 \star \star \star$ \\
\hline Q1 (lower) & 366 & $\begin{array}{c}5.3 \\
(4.9,5.7)\end{array}$ & $\begin{array}{c}17.2 \\
(17.0,17.5)\end{array}$ & 375 & $\begin{array}{c}20,8 \\
(19.9,21.2)\end{array}$ & $\begin{array}{c}75.7 \\
(74.8,76.5)\end{array}$ & 359 & $\begin{array}{c}3.7 \\
(3.4,3.9)\end{array}$ & $\begin{array}{c}12,2 \\
(12,1,12,3)\end{array}$ \\
\hline Q2 & 351 & $\begin{array}{c}6.0 \\
(5.5,6.4)\end{array}$ & $\begin{array}{c}17.6 \\
(17.3,17.8)\end{array}$ & 356 & $\begin{array}{c}22.6 \\
(21.7,23.6)\end{array}$ & $\begin{array}{c}73.9 \\
(73.0,74.9)\end{array}$ & 346 & $\begin{array}{c}4.0 \\
(3.7,4.3)\end{array}$ & $\begin{array}{c}12,2 \\
(12,1,12,2)\end{array}$ \\
\hline Q3 & 361 & $\begin{array}{c}6.4 \\
(6.0,6.9)\end{array}$ & $\begin{array}{c}17.7 \\
(17.5,17.9)\end{array}$ & 368 & $\begin{array}{c}24.0 \\
(23.1,24.9)\end{array}$ & $\begin{array}{c}72.5 \\
(71.7,73.4)\end{array}$ & 360 & $\begin{array}{c}4.4 \\
(4.1,4.6)\end{array}$ & $\begin{array}{c}12,2 \\
(12,1,12,3)\end{array}$ \\
\hline Q4 & 255 & $\begin{array}{c}7.4 \\
(6.8,7.9)\end{array}$ & $\begin{array}{c}18.2 \\
(17.9,18.5)\end{array}$ & 260 & $\begin{array}{c}25.7 \\
(24.5,26.8)\end{array}$ & $\begin{array}{c}71.0 \\
(69.8,72.1)\end{array}$ & 253 & $\begin{array}{c}4.8 \\
(4.5,5.2)\end{array}$ & $\begin{array}{c}12,2 \\
(12,1,12,3)\end{array}$ \\
\hline Q5 (upper) & 255 & $\begin{array}{c}7.0 \\
(6.5,7.6)\end{array}$ & $\begin{array}{c}18.0 \\
(17.8,18.3)\end{array}$ & 264 & $\begin{array}{c}25.2 \\
(24.0,26.3)\end{array}$ & $\begin{array}{c}71.4 \\
(70.3,72.5)\end{array}$ & 253 & $\begin{array}{c}4.6 \\
(4.3,5.0)\end{array}$ & $\begin{array}{c}12,2 \\
(12,0,12,3)\end{array}$ \\
\hline Maternal education (years) & & $p<0.0001 * *$ & $\mathrm{p}<0.0001$ * & & $p<0.0001 * \star$ & $p<0.0001 * \star$ & & $\mathrm{p}<0.0001$ ** & $\mathrm{p}=0,2^{\star \star \star}$ \\
\hline $0-4$ & 238 & $\begin{array}{c}5.5 \\
(4.9,6.0)\end{array}$ & $\begin{array}{c}17.3 \\
(17.0,17.7)\end{array}$ & 244 & $21.0(19.8,22.1)$ & $75.6(74.5,76.7)$ & 234 & $3.8(3.4,4.1)$ & $12,3(12,2,12,4)$ \\
\hline $5-8$ & 673 & $\begin{array}{c}6.0 \\
(5.7,6.3)\end{array}$ & $\begin{array}{c}17.5 \\
(17.3,17.7)\end{array}$ & 686 & $\begin{array}{c}22.9 \\
(22.2,23.6)\end{array}$ & $\begin{array}{c}73.6 \\
(72.9,74.3)\end{array}$ & 669 & $\begin{array}{c}4.1 \\
(3.9,4.3)\end{array}$ & $\begin{array}{c}12.1 \\
(12.1,12.2)\end{array}$ \\
\hline $9-11$ & 530 & $\begin{array}{c}6.7 \\
(6.4,7.1)\end{array}$ & $\begin{array}{c}18.0 \\
(17.8,18.1)\end{array}$ & 543 & $\begin{array}{c}24.5 \\
(23.7,25.3)\end{array}$ & $\begin{array}{c}72.1 \\
(71.4,72.9)\end{array}$ & 522 & $\begin{array}{c}4.5 \\
(4.3,4.7)\end{array}$ & $\begin{array}{c}12.2 \\
(12.1,12.3)\end{array}$ \\
\hline 12 or more & 136 & $\begin{array}{c}7.4 \\
(6.6,8.1)\end{array}$ & $18.3(18.0,18.7)$ & 138 & $\begin{array}{c}25.8 \\
(24.2,27.4)\end{array}$ & $\begin{array}{c}70.8 \\
(69.3,72.4)\end{array}$ & 135 & $\begin{array}{c}4.8 \\
(4.4,5.3)\end{array}$ & $\begin{array}{c}12.2 \\
(12.1,12.4)\end{array}$ \\
\hline Skin color & & $p=0.07 * \star \star$ & $p=0.6 * \star \star$ & & $p=0.002 * \star \star$ & $p=0.003 \star \star \star$ & & $p=0.04 * \star \star$ & $p=0.6^{\star * \star}$ \\
\hline White & 1,069 & $\begin{array}{c}6.5 \\
(6.2,6.7)\end{array}$ & $\begin{array}{c}17.6 \\
(17.5,17.8)\end{array}$ & 1,094 & $\begin{array}{c}24.0 \\
(23.4,24,5)\end{array}$ & $\begin{array}{c}72.6 \\
(72.1,73.2)\end{array}$ & 1,059 & $\begin{array}{c}4.4 \\
(4.2,4.5)\end{array}$ & $\begin{array}{c}12.2 \\
(12.1,12.2)\end{array}$ \\
\hline Black & 183 & $\begin{array}{c}5.7 \\
(5.1,6.3)\end{array}$ & $\begin{array}{c}17.9 \\
(17.5,18.2)\end{array}$ & 186 & $\begin{array}{c}21.4 \\
(20.1,22.7)\end{array}$ & $\begin{array}{c}75.1 \\
(73.8,76.3)\end{array}$ & 180 & $\begin{array}{c}3.8 \\
(3.5,4.2)\end{array}$ & $\begin{array}{c}12.3 \\
(12.1,12.4)\end{array}$ \\
\hline Brown & 231 & $\begin{array}{c}6.0 \\
(5.5,6.5)\end{array}$ & $\begin{array}{c}17.7 \\
(17.5,18.0)\end{array}$ & 237 & $\begin{array}{c}22.5 \\
(21.3,23.7)\end{array}$ & $\begin{array}{c}74.0 \\
(72.8,75.1)\end{array}$ & 228 & $\begin{array}{c}4.1 \\
(3.7,4.4)\end{array}$ & $\begin{array}{c}12.2 \\
(12.1,12.3)\end{array}$ \\
\hline Other & 98 & $\begin{array}{c}6.2 \\
(5.3,7.1)\end{array}$ & $\begin{array}{c}17.6 \\
(17.2,18.0)\end{array}$ & 99 & $\begin{array}{c}23.0 \\
(21.0,25.1)\end{array}$ & $\begin{array}{c}73.6 \\
(71.5,75.6)\end{array}$ & 97 & $\begin{array}{c}4.1 \\
(3.6,4.7)\end{array}$ & $\begin{array}{c}12.2 \\
(12.0,12.4)\end{array}$ \\
\hline Gestational age (weeks) & & $p=0.003 \#$ & $p=0.0005^{\star \star \star}$ & & $p=0.01 \star \star \star$ & $\mathrm{p}=0.01 \star \star \star$ & & $p=0.01 \#$ & $p=0.5^{\star \star \star}$ \\
\hline$<34$ & 32 & $\begin{array}{c}5.5 \\
(4.2,6.8)\end{array}$ & $\begin{array}{c}16.7 \\
(16.1,17.4)\end{array}$ & 32 & $\begin{array}{c}21.9 \\
(18.6,25.2)\end{array}$ & $\begin{array}{c}74.6 \\
(71.3,77.9)\end{array}$ & 31 & $\begin{array}{c}3.9 \\
(3.1,4.7)\end{array}$ & $\begin{array}{c}12.2 \\
(11.9,12.5)\end{array}$ \\
\hline $34-36$ & 169 & $\begin{array}{c}5.4 \\
(4.9,6.0)\end{array}$ & $\begin{array}{c}17.2 \\
(16.9,17.5)\end{array}$ & 171 & $\begin{array}{c}21.4 \\
(20.1,22.7)\end{array}$ & $\begin{array}{c}75.1 \\
(73.8,76.4)\end{array}$ & 167 & $\begin{array}{c}3.7 \\
(3.4,4.0)\end{array}$ & $\begin{array}{c}12.1 \\
(12.0,12.2)\end{array}$ \\
\hline $37-41$ & 1,287 & $\begin{array}{c}6.5 \\
(6.2,6.7)\end{array}$ & $\begin{array}{c}17.8 \\
(17.7,17.9)\end{array}$ & 1,318 & $\begin{array}{c}23.8 \\
(23.2,24.3)\end{array}$ & $\begin{array}{c}72.8 \\
(72.3,73.3)\end{array}$ & 1,273 & $\begin{array}{c}4.3 \\
(4.2,4.5)\end{array}$ & $\begin{array}{c}12.2 \\
(12.2,12.2)\end{array}$ \\
\hline$>41$ & 106 & $\begin{array}{c}6.0 \\
(5.2,6.7)\end{array}$ & $\begin{array}{c}17,4 \\
(17.0,17.8)\end{array}$ & 108 & $\begin{array}{c}22.7 \\
(20.9,24.5)\end{array}$ & $\begin{array}{c}73.8 \\
(72.0,75.5)\end{array}$ & 106 & $\begin{array}{c}4.1 \\
(3.6,4.5)\end{array}$ & $\begin{array}{c}12.1 \\
(12.0,12.3)\end{array}$ \\
\hline
\end{tabular}

* Test for linear trend (non-parametric);

** Test for linear trend (parametric);

*** Test of homogeneity (parametric);

\# Test of homogeneity (non-parametric)

with higher means seen among black ones 7,10. The present study found a mean difference of $0.2 \mathrm{~kg}$ and $0.3 \mathrm{~kg}$ of LBM between white and black boys and girls, respectively, but it was not statistically significant.
Mean adiposity measures showed a positive association with socioeconomic variables (socioeconomic index and maternal education level); higher means were seen in boys and girls from better-off families and born to mothers that were 
Table 5

Mean fat mass (FM) and mean lean body mass (LBM) in boys, according to the study independent variables. The 2004 Pelotas Birth Cohort. Pelotas, Rio Grande do Sul State, Brazil, 2013, 2013. (N=3,373).

\begin{tabular}{|c|c|c|c|c|c|c|c|c|c|}
\hline \multirow[t]{2}{*}{ Variables } & \multicolumn{3}{|c|}{$\mathrm{kg}$} & \multicolumn{3}{|c|}{$\%$} & \multicolumn{3}{|c|}{$\mathrm{kg} / \mathrm{m}^{2}$} \\
\hline & $\mathbf{n}$ & FM & LM & $\mathbf{n}$ & FM & LM & $\mathbf{n}$ & FM & LM \\
\hline Socioeconomic index & & $\mathrm{p}<0.0001$ * & $p<0.0001 * \star$ & & $p<0.0001$ * & $p<0.0001$ * & & $p<0.0001$ * & $p=0.8^{* \star \star}$ \\
\hline Q1 (lower) & 380 & $\begin{array}{c}3.6 \\
(3.3,3.9)\end{array}$ & $\begin{array}{c}18.6 \\
(18.4,18,9)\end{array}$ & 395 & $\begin{array}{c}14.3 \\
(13.6,15.0)\end{array}$ & $\begin{array}{c}82.1 \\
(81.4,82.8)\end{array}$ & 374 & $\begin{array}{c}2.5 \\
(2.3,2.6)\end{array}$ & $\begin{array}{c}13.0 \\
(13.0,13.1)\end{array}$ \\
\hline Q2 & 344 & $\begin{array}{c}4.5 \\
(4.1,4.9)\end{array}$ & $\begin{array}{c}19.2 \\
(19.0,19.5)\end{array}$ & 357 & $\begin{array}{c}16.6 \\
(15.7,17.5)\end{array}$ & $\begin{array}{c}79.8 \\
(78.9,80.7)\end{array}$ & 339 & $\begin{array}{c}3.0 \\
(2.7,3.2)\end{array}$ & $\begin{array}{c}13.0 \\
(12.9,13.2)\end{array}$ \\
\hline Q3 & 385 & $\begin{array}{c}5.2 \\
(4.9,5.6)\end{array}$ & $\begin{array}{c}19.3 \\
(19.0,19.5)\end{array}$ & 390 & $\begin{array}{c}18.8 \\
(17.9,19.8)\end{array}$ & $\begin{array}{c}77.7 \\
(76.8,78.6)\end{array}$ & 379 & $\begin{array}{c}3.4 \\
(3.2,3.7)\end{array}$ & $\begin{array}{c}13.0 \\
(12.9,13.1)\end{array}$ \\
\hline Q4 & 288 & $\begin{array}{c}5.4 \\
(5.0,5.9)\end{array}$ & $\begin{array}{c}19.6 \\
(19.3,19.8)\end{array}$ & 291 & $\begin{array}{c}19.2 \\
(18.1,20.3)\end{array}$ & $\begin{array}{c}77.3 \\
(76.2,78.3)\end{array}$ & 286 & $\begin{array}{c}3.5 \\
(3.2,3.8)\end{array}$ & $\begin{array}{c}13.0 \\
(12.9,13.1)\end{array}$ \\
\hline Q5 (upper) & 297 & $\begin{array}{c}6.6 \\
(6.1,7.1)\end{array}$ & $\begin{array}{c}20.0 \\
(19.7,20.2)\end{array}$ & 304 & $\begin{array}{c}22.1 \\
(21.0,23.2)\end{array}$ & $\begin{array}{c}74.5 \\
(73.4,75.5)\end{array}$ & 295 & $\begin{array}{c}4.2 \\
(3.9,4.5)\end{array}$ & $\begin{array}{c}13.0 \\
(12.9,13.1)\end{array}$ \\
\hline Maternal education (years) & & $p<0.0001$ * & $\mathrm{p}<0.0001 * \star$ & & $\mathrm{p}<0.0001$ * & $p<0.0001$ * & & $\mathrm{p}<0.0001$ * & $p=0.7 \star \star \star$ \\
\hline $0-4$ & 255 & $\begin{array}{c}3.7 \\
(3.3,4.1)\end{array}$ & $\begin{array}{c}18.7 \\
(18.4,18.9)\end{array}$ & 260 & $\begin{array}{c}14.7 \\
(13.8,15.6)\end{array}$ & $\begin{array}{c}81.8 \\
(80.9,82.6)\end{array}$ & 251 & $\begin{array}{c}2.5 \\
(2.3,2.7)\end{array}$ & $\begin{array}{c}13.0 \\
(12.9,13.1)\end{array}$ \\
\hline $5-8$ & 690 & $\begin{array}{c}4.5 \\
(4.2,4.7)\end{array}$ & $\begin{array}{c}19.1 \\
(18.9,19.2)\end{array}$ & 714 & $\begin{array}{c}16.7 \\
(16.1,17.3)\end{array}$ & $\begin{array}{c}79.7 \\
(79.1,80.4)\end{array}$ & 679 & $\begin{array}{c}3.0 \\
(2.8,3.1)\end{array}$ & $\begin{array}{c}13.0 \\
(13.0,13.1)\end{array}$ \\
\hline $9-11$ & 570 & $\begin{array}{c}5.9 \\
(5.5,6.2)\end{array}$ & $\begin{array}{c}19.7 \\
(19.5,19.9)\end{array}$ & 578 & $\begin{array}{c}20.3 \\
(19.5,21.1)\end{array}$ & $\begin{array}{c}76.2 \\
(75.4,77.0)\end{array}$ & 565 & $\begin{array}{c}3.8 \\
(3.6,4.0)\end{array}$ & $\begin{array}{c}13.0 \\
(12.9,13.1)\end{array}$ \\
\hline 12 or more & 175 & $\begin{array}{c}5.9 \\
(5.3,6.5)\end{array}$ & $\begin{array}{c}19.9 \\
(19.5,20.2)\end{array}$ & 180 & $\begin{array}{c}20.2 \\
(18.9,21.6)\end{array}$ & $\begin{array}{c}76.3 \\
(75.0,77.6)\end{array}$ & 174 & $\begin{array}{c}3.7 \\
(3.4,4.1)\end{array}$ & $\begin{array}{c}13.0 \\
(12.8,13.1)\end{array}$ \\
\hline Skin color & & $p=0.0001 \#$ & $p=0.3 * \star \star$ & & $p=0.2 * \star \star$ & 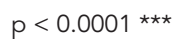 & & $p=0.0001 \#$ & $p=0.02 * \star \star$ \\
\hline White & 1,122 & $\begin{array}{c}5.2 \\
(5.0,5.4)\end{array}$ & $\begin{array}{c}19.3 \\
(19.1,19.4)\end{array}$ & 1150 & $\begin{array}{c}18.9 \\
(18.3,19.4)\end{array}$ & $\begin{array}{c}77.7 \\
(77.1,78.2)\end{array}$ & 1,109 & $\begin{array}{c}3.4 \\
(3.3,3.5)\end{array}$ & $\begin{array}{c}13.0 \\
(12.9,13.0)\end{array}$ \\
\hline Black & 225 & $\begin{array}{c}4.4 \\
(3.8,4.9)\end{array}$ & $\begin{array}{c}19.5 \\
(19.2,19.8)\end{array}$ & 231 & $\begin{array}{c}15.3 \\
(14.1,16.5)\end{array}$ & $\begin{array}{c}81.1 \\
(79.9,82.3)\end{array}$ & 221 & $\begin{array}{c}2.8 \\
(2.5,3.2)\end{array}$ & $\begin{array}{c}13.1 \\
(13.0,13.2)\end{array}$ \\
\hline Brown & 245 & $\begin{array}{c}4.7 \\
(4.2,5.2)\end{array}$ & $\begin{array}{c}19.1 \\
(18.8,19.4)\end{array}$ & 252 & $\begin{array}{c}16.9 \\
(15.8,18.1)\end{array}$ & $\begin{array}{c}79.5 \\
(78.3,80.6)\end{array}$ & 243 & $\begin{array}{c}3.1 \\
(2.8,3.4)\end{array}$ & $\begin{array}{c}13.1 \\
(13.0,13.2)\end{array}$ \\
\hline Other & 81 & $\begin{array}{c}4.4 \\
(3.6,5.1)\end{array}$ & $\begin{array}{c}19.2 \\
(18.7,19.7)\end{array}$ & 82 & $\begin{array}{c}16.4 \\
(14.7,18.1)\end{array}$ & $\begin{array}{c}80.0 \\
(78.3,81.7)\end{array}$ & 80 & $\begin{array}{c}2.9 \\
(2.5,3.3)\end{array}$ & $\begin{array}{c}13 \cdot 1 \\
(12,9 \cdot 13,3)\end{array}$ \\
\hline Gestational age (weeks) & & $\mathrm{p}=0.001 \star \star \star \star$ & $p<0.0001 * \star *$ & & $p=0.3 * \star \star$ & $p<0.0001 * \star \star$ & & $\mathrm{p}=0.0007 \star \star \star \star$ & $\mathrm{p}=0.002^{\star \star \star}$ \\
\hline$<34$ & 36 & $\begin{array}{c}3.7 \\
(2.5,4.9)\end{array}$ & $\begin{array}{c}18.0 \\
(17.1,18.9)\end{array}$ & 39 & $\begin{array}{c}14.1 \\
(11.5,16.7)\end{array}$ & $\begin{array}{c}82.2 \\
(79.7,84.7)\end{array}$ & 36 & $\begin{array}{c}2.4 \\
(1.8,3.1)\end{array}$ & $\begin{array}{c}12.7 \\
(12.5,13.0)\end{array}$ \\
\hline $34-36$ & 172 & $\begin{array}{c}4.1 \\
(3.5,4.7)\end{array}$ & $\begin{array}{c}18.8 \\
(18.4,19.2)\end{array}$ & 174 & $\begin{array}{c}15.5 \\
(14.3,16.8)\end{array}$ & $\begin{array}{c}80.9 \\
(79.6,82.1)\end{array}$ & 169 & $\begin{array}{c}2.7 \\
(2.4,3.1)\end{array}$ & $\begin{array}{c}12.8 \\
(12.7,12.9)\end{array}$ \\
\hline $37-41$ & 1,386 & $\begin{array}{c}5.2 \\
(4.9,5.4)\end{array}$ & $\begin{array}{c}19.4 \\
(19.3,19.5)\end{array}$ & 1,421 & $\begin{array}{c}18.5 \\
(18.0,18.9)\end{array}$ & $\begin{array}{c}78.0 \\
(77.6,78.5)\end{array}$ & 1,370 & $\begin{array}{c}3.4 \\
(3.2,3.5)\end{array}$ & $\begin{array}{c}13.1 \\
(13.0,13.1)\end{array}$ \\
\hline$>41$ & 105 & $\begin{array}{c}4.5 \\
(3.9,5.2)\end{array}$ & $\begin{array}{c}18.9 \\
(18.5,19.3)\end{array}$ & 108 & $\begin{array}{c}16.9 \\
(15.1,18.6)\end{array}$ & $\begin{array}{c}79.6 \\
(77.9,81.2)\end{array}$ & 103 & $\begin{array}{c}3.0 \\
(2.6,3.4)\end{array}$ & $\begin{array}{c}13.0 \\
(12.8,13.2)\end{array}$ \\
\hline
\end{tabular}

* Test for linear trend (non-parametric);

** Test for linear trend (parametric);

*** Test of homogeneity (parametric);

\# Test of homogeneity (non-parametric). 
more educated. Mean LBM also increased with socioeconomic index and maternal education level; however, after adjusting for height, these differences disappeared. These results suggest that, similar to LBM, height also increased with socioeconomic index and maternal education levels in both boys and girls. A study with children from the Soweto birth cohort in South Africa 19 found only an association of mean LBM with maternal marital status (higher mean LBM in children of mothers living with a partner). But children from households that owned television sets and a car and in which no family member was unemployed showed higher mean FMI in late childhood 19 .

Socioeconomic conditions assessed through household income did not show any association with FM in US children at age five 7 . However, studies that used type of parental employment to assess socioeconomic level found higher mean adiposity measures and fat-free mass among children of parents with less specialized-skill occupations 14,20. Studies that used parental education level as a socioeconomic indicator found different associations with adiposity measures during childhood: a negative association with maternal and paternal education level 16; a negative association with paternal education only 20 ; and lack of association 7. When assessed, LBM measures did not show any association with socioeconomic indicators 20.

Mean adiposity measures were significantly lower among preterm children. An association between prematurity and mean LBM was seen in boys and girls, but it remained significant only in boys after adjusting for height. Means in late preterm (34 to 36 weeks of gestation) for boys and girls were closer to those seen in preterm children born at less than 34 weeks of gestation than to those seen in full-term children, which is consistent with a disadvantaged nutritional status described in these children ${ }^{36}$. It is well established that preterm children have lower levels of FM at birth 13 , a state that can be attributed to reduced fat deposition which occurs largely in the last trimester of pregnancy. This reduced fat content seems to persist throughout childhood 37 . Magnetic resonance imaging studies have suggested that preterm children have a more central distribution of fat tissue 38 suggesting that this state persists in later years 39 .

In conclusion, despite of the fact that Brazil is in epidemiological and nutritional transitions, this study confirms the difference between child sexes and emphasizes the important role of skin color over body composition as reported from other countries. Although prevalence of overweight/obesity ( $>+1$ SD of BMI Z-score) is high among children from all SES stratum $(25 \%$ among the poorest and $48 \%$ among the richest from the 2004 Pelotas Birth Cohort) the finding of this study raises the hypothesis that better socioeconomic conditions may contribute to increased adiposity in childhood. There is still a dearth of studies concerned with the longterm consequences of body composition due to prematurity. 


\section{Resumen}

El objetivo de este estudio fue describir la masa grasa (MG) y la masa libre de grasa (MLG), estratificadas por sexo en niños de seis años de edad, dentro de una cohorte de nacimientos de 2004 en Pelotas,. La absorciometría de doble fotón (DXA por sus siglas en inglés) fue utilizada para medir la MG y la MLG, los indices de MG y MLG, y el porcentaje (\%) de MG y MLG. Las medias de adiposidad fueron mayores entre las niñas $(6,3 \mathrm{~kg}$, $4,2 \mathrm{~kg} / \mathrm{m}^{2}$ y $23,4 \%$ vs. $5,0 \mathrm{~kg}, 3,3 \mathrm{~kg} / \mathrm{m}^{2}$ y $18 \%$ ), mientras que las medidas de MLG fueron mayores entre los niños (19,3kg, $13 \mathrm{~kg} / \mathrm{m}^{2}$ y $78,5 \%$ vs. $17,7 \mathrm{~kg}, 12,2 \mathrm{~kg} / \mathrm{m}^{2}$ y 73,2\%). En niños y niñas, las medias de adiposidad aumentan con el nivel socioeconómico y la educación materna. Las medias de adiposidad fueron mayores entre niños de piel blanca, mientras que el \%MLG fue mayor entre los de piel negra. Los prematuros presentaron medias más bajas de adiposidad y MLG, en comparación con el resto. El sexo femenino, color de piel blanca y condiciones socioeconómicas más altas están asociados a una mayor adiposidad en la niñez.

Composición Corporal; Niño; Estudios Longitudinales

\section{Contributors}

R. V. Zanini designed and developed this research study, conducted data collection, analyzed the data, prepared the manuscript draft, was responsible for reviewing its content and read and approved the final manuscript. I. S. Santos designed and developed this research study, conducted data collection, prepared the manuscript draft and read and approved the final manuscript. D. P. Gigante designed and developed this research study, prepared the manuscript draft and read and approved the final manuscript. A. Matijasevich, F. C. Barros and A. J. D. Barros conducted data collection and read and approved the final manuscript. F. C. Barros conducted data collection and read and approved the final manuscript.

\section{Acknowledgments}

This article is based on data from the Pelotas Birth Cohort, 2004 study conducted by the Postgraduate Program in Epidemiology at the Universidade Federal de Pelotas. The 2004 birth cohort study is currently supported by the Wellcome Trust through the program entitled Major Awards for Latin America on Health Consequences of Population Change. The World Health Organization, National Support Program for Centers of Excellence (PRONEX), Brazilian National Research Council (CNPq), Brazilian Ministry of Health and the Children's Pastorate supported previous phases of the study.

\section{References}

1. Ellis KJ. Human body composition: in vivo methods. Physiol Rev 2000; 80:649-80.

2. Sopher A, Shen W, Pietrobelli A. Pediatric Body Composition Methods. In: Heymsfield SB, Lohman TG, Wang Z, Going SB, editors. Human body composition. 2nd Ed. Champaign: Human Kinetics; 2005. p. 129-40.

3. Martin AD, Drinkwater DT. Variability in the measures of body fat. Assumptions or technique? Sports Med 1991; 11:277-88.

4. Ay L, Van Houten VA, Steegers EA, Hofman A, Witteman JC, Jaddoe VW, et al. Fetal and postnatal growth and body composition at 6 months of age. J Clin Endocrinol Metab 2009; 94:2023-30.
5. Henche SA, Torres RR, Pellico LG. An evaluation of patterns of change in total and regional body fat mass in healthy Spanish subjects using dual-energy X-ray absorptiometry (DXA). Eur J Clin Nutr 2008; 62:1440-8.

6. Robinson SM, Marriott LD, Crozier SR, Harvey NC, Gale CR, Inskip HM, et al. Variations in infant feeding practice are associated with body composition in childhood: a prospective cohort study. J Clin Endocrinol Metab 2009; 94:2799-805.

7. Burdette HL, Whitaker RC, Hall WC, Daniels SR. Breastfeeding, introduction of complementary foods, and adiposity at $5 \mathrm{y}$ of age. Am J Clin Nutr 2006; 83:550-8. 
8. Lim JS, Hwang JS, Cheon GJ, Lee JA, Kim DH, Park $\mathrm{KD}$, et al. Gender differences in total and regional body composition changes as measured by dualenergy x-ray absorptiometry in Korean children and adolescents. J Clin Densitom 2009; 12:229-37.

9. Taylor RW, Grant AM, Williams SM, Goulding A. Sex differences in regional body fat distribution from pre- to postpuberty. Obesity (Silver Spring) 2010; 18:1410-6.

10. Borrud LG, Flegal KM, Looker AC, Everhart JE, Harris TB, Shepherd JA. Body composition data for individuals 8 years of age and older: U.S. population, 1999-2004. Vital Health Stat 2010; (250):1-87.

11. Mueller WH, Harrist RB, Doyle SR, Labarthe DR. Percentiles of body composition from bioelectrical impedance and body measurements in U.S. adolescents 8-17 years old: Project HeartBeat! Am J Hum Biol 2004; 16:135-50.

12. Wells JC, Hallal PC, Wright A, Singhal A, Victora CG. Fetal, infant and childhood growth: relationships with body composition in Brazilian boys aged 9 years. Int J Obes (Lond) 2005; 29:1192-8.

13. Carberry AE, Colditz PB, Lingwood BE. Body composition from birth to 4.5 months in infants born to non-obese women. Pediatr Res 2010; 68:84-8.

14. Chomtho S, Wells JC, Williams JE, Lucas A, Fewtrell MS. Associations between birth weight and later body composition: evidence from the 4-component model. Am J Clin Nutr 2008; 88:1040-8.

15. Dixon B, Pena MM, Taveras EM. Lifecourse approach to racial/ethnic disparities in childhood obesity. Adv Nutr 2012; 3:73-82.

16. Schnurbein J, Klenk J, Galm C, Berg S, Gottmann P, Steinacker JM, et al. Reference values and early determinants of intra-abdominal fat mass in primary school children. Horm Res Paediatr 2011; 75: 412-22.

17. Huus K, Ludvigsson JF, Enskar K, Ludvigsson J. Exclusive breastfeeding of Swedish children and its possible influence on the development of obesity: a prospective cohort study. BMC Pediatr 2008; 8:42.

18. Durmus B, Ay L, Duijts L, Moll HA, Hokken-Koelega AC, Raat $\mathrm{H}$, et al. Infant diet and subcutaneous fat mass in early childhood: the Generation $\mathrm{R}$ Study. Eur J Clin Nutr 2012; 66:253-60.

19. Griffiths PL, Rousham EK, Norris SA, Pettifor JM, Cameron N. Socio-economic status and body composition outcomes in urban South African children. Arch Dis Child 2008; 93:862-7.

20. Boot AM, Bouquet J, de Ridder MA, Krenning EP, de Muinck Keizer-Schrama SM. Determinants of body composition measured by dual-energy Xray absorptiometry in Dutch children and adolescents. Am J Clin Nutr 1997; 66:232-8.

21. Batista Filho M, Rissin A. A transição nutricional no Brasil: tendências regionais e temporais. Cad Saúde Pública 2003; 19 Suppl 1:S181-S91.

22. Schmidt MI, Duncan BB, Azevedo e Silva G, Menezes AM, Monteiro CA, Barreto SM, et al. Chronic non-communicable diseases in Brazil: burden and current challenges. Lancet 2011;377:1949-61.
23. Santos IS, Barros AJ, Matijasevich A, Domingues MR, Barros FC, Victora CG. Cohort profile: The 2004 Pelotas (Brazil) Birth Cohort Study. Int J Epidemiol 2011; 40:1461-8.

24. Wells JC, Cole TJ. Adjustment of fat-free mass and fat mass for height in children aged $8 \mathrm{y}$. Int J Obes Relat Metab Disord 2002; 26:947-52.

25. Habicht JP. Estandarización de métodos epidemiológicos cuantitativos sobre el terreno. Bol Oficina Sanit Panam 1974; 76:375-84

26. Barros AJD, Victora CG. Indicador econômico para o Brasil baseado no censo demográfico de 2000. Rev Saúde Pública 2005; 39:523-9.

27. Martin JA, Hamilton BE, Sutton PD, Ventura SJ, Menacker F, Munson ML. Births: final data for 2003. Natl Vital Stat Rep 2005;54(2):1-116.

28. Fenton TR. A new growth chart for preterm babies: Babson and Benda's chart updated with recent data and a new format. BMC Pediatr 2003; 3:13.

29. Dubowitz LM, DubowitzV, Goldberg C. Clinical assessment of gestational age in the newborn infant. J Pediatr 1970; 77:1-10

30. Lohman TG, Chen Z. Dual-Energy X-Ray Absorptiometry. In: Heymsfield SB, Lohman TG, Wang Z, Going SB, editors. Human body composition. 2nd Ed. Champaign: Human Kinetics; 2005. p. 63-78.

31. Freedman DS, Wang J, Thornton JC, Mei Z, Pierson RN, Jr., Dietz WH, et al. Racial/ethnic differences in body fatness among children and adolescents. Obesity (Silver Spring). 2008 May;16(5):1105-11.

32. Huang TT, Johnson MS, Figueroa-Colon R, Dwyer JH, Goran MI. Growth of visceral fat, subcutaneous abdominal fat, and total body fat in children. Obes Res 2001; 9:283-9.

33. Baumgartner RN. Age. In: Heymsfield SB, Lohman TG, Wang Z, Going SB, editors. Human body composition. 2nd Ed. Champaign: Human Kinetics; 2005. p. 259-70.

34. McCarthy HD, Cole TJ, Fry T, Jebb SA, Prentice AM. Body fat reference curves for children. Int J Obes 2006; 30:598-602.

35. Ma J, Feng N, Zhang SW, Pan YP, Huang YB. Comparison of changes in body composition during puberty development of obese and normal-weight children in China. Biomed Environ Sci 2009; 22:413-8.

36. Santos IS, Matijasevich A, Domingues MR, Barros AJ, Victora CG, Barros FC. Late preterm birth is a risk factor for growth faltering in early childhood: a cohort study. BMC Pediatr 2009; 9:71

37. Fewtrell MS, Lucas A, Cole TJ, Wells JC. Prematurity and reduced body fatness at 8-12 y of age. Am J Clin Nutr 2004; 80:436-40.

38. Uthaya S, Thomas EL, Hamilton G, Dore CJ, Bell J, Modi N. Altered adiposity after extremely preterm birth. Pediatr Res 2005; 57:211-5.

39. Wells JC, Chomtho S, Fewtrell MS. Programming of body composition by early growth and nutrition. Proc Nutr Soc 2007; 66:423-34.

Submitted on 23/Aug/2013

Final version resubmitted on 27/Dec/2013 Approved on 17/Feb/2014 